\title{
Focused ultrasound is the next step in perioperative care
}

\author{
André Denault, MD, PhD • Ashraf Fayad, MD • \\ Robert Chen, MD
}

Received: 25 February 2013/Accepted: 7 May 2013/Published online: 21 May 2013

(C) Canadian Anesthesiologists' Society 2013

In this issue of the Journal, two different investigators report the use of bedside ultrasound for focused cardiac examination as well as for focused thoracic and abdominal examinations in patients undergoing non-cardiac surgery. ${ }^{1,2}$ These ultrasound observations led to a change in patient management, and it is expected that more widespread use of this tool in our practice will likely have a significant impact on perioperative care. This paradigm change implies that consideration should be given to incorporating an ultrasound curriculum within our anesthesiology training programs. ${ }^{3}$

The first case ${ }^{1}$ involves a previously healthy 29 -yr-old pregnant woman who developed preeclampsia complicated by HELLP (hemolysis, elevated liver enzymes, and low platelet count) syndrome. Following Cesarean delivery, the patient remained anuric and was transferred to the intensive care unit. A bedside cardiac ultrasound was performed using a validated pulsed wave tissue Doppler technique to estimate filling pressures and evaluate cardiac function. ${ }^{4}$ The patient was found to be responsive to passive leg raising and fluid therapy, and ultrasound confirmed the

\section{A. Denault, $\mathrm{MD}, \mathrm{PhD}(\bowtie)$}

Department of Anesthesia, Montreal Heart Institute, 5000 Belanger Street, Montreal, QC H1S 1T8, Canada e-mail: andre.denault@gmail.com

\section{A. Denault, $\mathrm{MD}, \mathrm{PhD}$}

Université de Montréal, Montreal, QC, Canada

\section{A. Fayad, MD}

Department of Anesthesia, Ottawa Civic Hospital, University of Ottawa, Ottawa, ON, Canada

R. Chen, MD

Department of Anesthesia, St. Michael's Hospital, University of Toronto, Toronto, ON, Canada absence of fluid overload; however, a few hours later, she was found to be anemic. At that point, abdominal ultrasound was used to diagnose an intra-abdominal hematoma. Based on these findings, a decision was made to transfuse blood and explore the abdomen surgically. Postoperatively, the patient became hypoxic, and a repeated lung ultrasound showed comet-tail artifacts of B-lines, which is suggestive of an alveolar-interstitial syndrome. The absence of elevated filling pressure on cardiac ultrasound suggested non-cardiogenic pulmonary edema and the possibility of acute respiratory distress syndrome.

The other case $^{2}$ involves a 58 -yr-old man who was scheduled for elective nasal polyp excision. The patient was known to have aortic stenosis and was "cleared" preoperatively by a cardiologist and a cardiac surgeon. A preoperative anesthesia evaluation of cardiac function with bedside ultrasound revealed an unsuspected large pericardial effusion with signs of pericardial tamponade and right atrial and right ventricular collapse. The case was cancelled and the patient underwent percutaneous pericardial drainage. The clinical presentation and pericardial fluid analysis led to the diagnosis of ChurgStrauss syndrome complicated by progressive pericardial effusion. The patient was discharged from hospital several days later, and his nasal polyps were treated medically.

These two cases effectively illustrate the gradual integration of bedside ultrasound as a point-of-care examination performed by the anesthesiologist for conditions that are not uncommon in the perioperative setting. ${ }^{5}$ Ultrasound was used in a goal-oriented approach to answer a specific question, and it involved more than solely examination of the heart. As anesthesiologists and critical care physicians, we strive to maintain adequate oxygen delivery; ultrasound can play a role in identifying 
conditions that may interfere with tissue oxygenation, such as hemodynamic instability, hypoxemia, and reduced urine output.

\section{Hemodynamic instability}

Bedside ultrasound allows prompt diagnosis of hemodynamic instability and the ability to repeat the exam as needed to assess response to therapy or rule out additional pathology. Cardiac output does not depend only on filling pressures. According to the concept developed by Guyton to explain shock states, at steady state, cardiac output equals venous return, which in turn is determined by three variables: mean systemic venous pressure, right atrial pressure, and resistance to venous return. ${ }^{6}$ Consequently, hemodynamic instability results from one of three distinct mechanisms or any combination of these. Point-of-care bedside ultrasound allows the identification of the mechanism(s) in question. The Table classifies the echocardiographic findings of the two case reports ${ }^{1,2}$ according to the mechanisms involved. The first patient ${ }^{1}$ initially presented with a decrease in mean systemic venous pressure indicative of distributive or hypovolemic shock due to hemorrhage. In contrast, the second patient ${ }^{2}$ had both left ventricular systolic and diastolic dysfunction combined with tamponade which led to an increase in right atrial pressure. Resistance to venous return was observed in both cases. In the patient with preeclampsia, ${ }^{1}$ extrinsic compression and development of an abdominal compartment syndrome ${ }^{7}$ due to uterine hematoma led to hemodynamic instability and obstructive shock. In the patient with pericardial effusion, obstructive shock was due to cardiac tamponade. Many of the above conditions could not have been diagnosed based on filling pressures alone, and ultrasound provided the best monitoring and diagnostic tool in these life-threatening cases.

\section{Hypoxemia}

Bedside ultrasound can be used to determine the etiology of hypoxia in more than $90 \%$ of cases. ${ }^{8}$ The use of lung ultrasound by anesthesiologists in the operating room was summarized in a recent editorial, ${ }^{9}$ but the technique is not new; it was described in detail in the critical care literature as early as two decades ago. ${ }^{10}$ Although lung ultrasound has been embraced as a useful technique in emergency medicine, it is still not widely taught in our anesthesiology or critical care training programs. Technical details on the use of this technique can be found in key review $\operatorname{articles}^{11,12}$ and are beyond the scope of this editorial. Nevertheless, understanding the mechanism of hypoxemia helps recognize the limitations of lung ultrasound. Basically, there are six mechanisms of hypoxemia or desaturation: pulmonary or extrapulmonary shunt, ventilation/perfusion mismatch, decreased diffusion, low inspired oxygen fraction, mixed venous oxygen desaturation, and hypoventilation. ${ }^{13}$ In the first patient, the authors initially mentioned the presence of a normal lung profile with A-lines and sliding lung (visceral pleura moving on the parietal pleura). The sliding lung sign rules out a pneumothorax. Later, B-lines or "comet tail" artifacts were observed. These B-lines are produced by the fluid-air interface; they are indicative of alveolar-interstitial syndrome and may be due to cardiogenic or noncardiogenic pulmonary edema. Therefore, it is important to combine both cardiac and pulmonary ultrasound findings to rule out one or the other etiology. Filling pressures were estimated using the Doppler mode, and initially, they were found to be within the normal range when A-lines were seen. Then filling pressures increased, and B-lines appeared due to both left ventricular systolic and diastolic dysfunction or as an early manifestation of acute respiratory distress syndrome. Not all mechanisms of hypoxia can be addressed with lung ultrasound, and some cases, such as intracardiac shunting, may also require cardiac ultrasound. Also, hypoxia due to low inspired fraction of oxygen or hypoventilation is diagnosed by vigilant clinical observation rather than by skilled ultrasound examination.

\section{Decreased urine output}

Decreased urine output can result from a pre-renal, renal, or post-renal cause, or a combination of those factors. Postrenal problems can be examined at the bedside by scanning

Table Role of bedside ultrasound in the identification of variables involved in oxygen delivery

\begin{tabular}{|c|c|c|c|}
\hline $\begin{array}{l}\text { Oxygen } \\
\text { arterial } \\
\text { content }\end{array}$ & $\begin{array}{l}\text { a-Decreased hemoglobin: abdominal } \\
\text { free fluid detection consistent with } \\
\text { bleeding. }\end{array}$ & $\begin{array}{l}\text { b-Decreased oxygen saturation: B-lines } \\
\text { or comet-tail artifacts consistent with } \\
\text { pulmonary edema }{ }^{1}\end{array}$ & \\
\hline $\begin{array}{l}\text { Cardiac } \\
\text { output or } \\
\text { venous } \\
\text { return }\end{array}$ & $\begin{array}{l}\text { a-Low mean venous systemic pressure: } \\
\text { abdominal free fluid detection } \\
\text { consistent with reduced volume. }\end{array}$ & $\begin{array}{l}\text { b-Increased right atrial pressure: left } \\
\text { ventricular systolic and diastolic } \\
\text { dysfunction }^{1} \text { and aortic stenosis }\end{array}$ & $\begin{array}{l}\text { c-Resistance to venous return: abdominal } \\
\text { compartment syndrome from uterine } \\
\text { hematoma }^{1} \text { and pericardial tamponade }\end{array}$ \\
\hline
\end{tabular}


the kidneys and the bladder to rule out hydronephrosis or a distended bladder due to a misplaced or kinked urinary catheter. Pre-renal involvement is assessed by investigating the causes of hemodynamic instability, as was the case in the first patient. ${ }^{1}$ Renal causes, such as interstitial nephritis, glomerulonephritis, and acute tubular necrosis may not be accessible by ultrasound technology. An important and often unrecognized cause of reduced urine output is abdominal hypertension, which can be due to abdominal compartment syndrome. ${ }^{7}$ This occurred in the first patient who developed a large intra-abdominal hematoma. ${ }^{1}$

\section{Preoperative evaluation}

With the more widespread adoption of hand-held devices, ultrasound will gradually be integrated as an extension of a patient's history and physical examination, as illustrated by the patient with the history of aortic stenosis. ${ }^{2}$ In this case, the ultrasound examination revealed an unsuspected pericardial effusion. Ultrasound has been reported as a complementary tool during preoperative evaluation not only to assess cardiac function but also to detect pulmonary edema, identify a pleural effusion, examine the airway, ${ }^{14}$ evaluate stomach content, ${ }^{15}$ assess diaphragmatic motion, ${ }^{16}$ and rule out deep vein thrombosis. ${ }^{17}$ In a retrospective study, a group based in Melbourne Australia showed that patients admitted with hip fractures who underwent a focused transthoracic ultrasound had lower mortality than a control group. ${ }^{18}$ Prospective studies should be undertaken to confirm these findings in similar settings.

\section{Limitations and training issues}

Bedside ultrasound complements but does not replace clinical judgement and it has its limitations. When the operator focuses his/her attention on the ultrasound images, more basic information, such as blood pressure and oxygen saturation, should not be neglected. Also, there are no alarms on an ultrasound machine, and the interpretation of images is operator-dependent. Consequently, the usefulness and impact of this tool depend not only on the quality of training but also on the number of ultrasound exams performed during training. Several societies have developed training guidelines to ensure competency. In critical care, the American College of Chest Physicians and the Société de Réanimation de Langue Française published such guidelines in 2009. ${ }^{19}$ This was followed by the first examination in critical care ultrasound in 2011. In a recent 2012 review, it was suggested to extend point-of-care ultrasound exams to many situations in anesthesiology. ${ }^{20}$ So far, most guidelines have been developed for cardiologists, emergency physicians, cardiac anesthesiologists, and critical care physicians. There are no clear training guidelines for non-cardiac anesthesiologists. In Canada, the Canadian Emergency Ultrasound Society (CEUS) (www.ceus.ca) provides training certification mostly for emergency physicians. In Ontario, practitioners must be certified by CEUS for billing purposes. The Cardiovascular and Thoracic Section of the Canadian Anesthesiologists' Society is currently developing a curriculum through the Royal College to address the issue of subspecialty certification for fellows in cardiac anesthesia. In addition, our group comprised of the Cardiovascular Thoracic, Perioperative Medicine, and Perioperative Echocardiography Sections is collaborating to develop guidelines for perioperative echocardiography in non-cardiac settings. The need for adequate training, certification, and education for basic and advanced training in anesthesiology will become an important issue in the coming years.

Anesthesiologists at the University of Melbourne have developed online courses leading to certification in bedside ultrasound. With the growing demands for this technology, ultrasound education may need to be considered at an earlier stage of anesthesia training. Several medical schools are now offering an ultrasound curriculum at the undergraduate level. ${ }^{21}$ At present, bedside decisions in many intensive care units worldwide are routinely made based on ultrasound-guided physical examination, and this was not the case ten years ago. ${ }^{22}$ Ultrasound is already present in the operating room to clarify causes of hemodynamic instability, hypoxemia, and oliguria/anuria, and with miniaturization and reduced cost, its use will likely spread to all operating rooms. Thus, guidelines are needed for perioperative ultrasound outside the cardiac operating rooms. The logical next step is to train ourselves and teach our residents in the use of this technology for elective and urgent scenarios, and numerous studies on how to apply this technology will appear in future. A parallel can be made with echoguided vascular access. A few years ago, this technique appeared as an optional variant that appealed to technological enthusiasts. Now, several studies have shown that echoguided vascular access has a higher success rate than the traditional landmark technique; it results in fewer complications and takes less time. ${ }^{23}$ So far, the advantages of bedside ultrasound have not been established with such a high quality of evidence, inasmuch as its impact is being documented only in case reports, such as those in this issue of the Journal. It is important that the impact and limitations of this new approach be evaluated in well-designed large clinical trials, as ultrasound is becoming both an addition to the anesthesia machine and an extension to the physical examination. 


\section{L'échographie ciblée: la prochaine étape dans les soins périopératoires}

Dans ce numéro du Journal, deux chercheurs distincts discutent de l'utilisation de l'échographie au chevet du patient pour réaliser un examen cardiaque ciblé ainsi que des examens thoraciques et abdominaux ciblés chez des patients subissant des chirurgies non cardiaques. ${ }^{1,2}$ Les résultats de ces examens échographiques ont entraîné des modifications dans la prise en charge des patients. Une utilisation plus répandue de cet outil dans notre pratique aura probablement un impact majeur sur les soins périopératoires. En raison de ce changement de paradigme, il convient de réfléchir à l'intégration possible d'un cours d'échographie dans nos programmes de formation en anesthésiologie. ${ }^{3}$

Le premier cas décrit ${ }^{1}$ est celui d'une femme enceinte de 29 ans auparavant en bonne santé qui a manifesté une pré-éclampsie, laquelle a été compliquée par un syndrome HELLP (hémolyse, augmentation des enzymes hépatiques, et numération plaquettaire faible). Après un accouchement par césarienne, la patiente est demeurée anurique et a été transférée à l'unité des soins intensifs. Une échographie cardiaque au chevet a été réalisée et une technique validée de Doppler tissulaire a été utilisée pour évaluer les pressions de remplissage et la fonction cardiaque. ${ }^{4} \mathrm{La}$ patiente a bien réagi à l'élévation passive de ses jambes et à la thérapie liquidienne, et l'échographie a confirmé l'absence de surcharge liquidienne; toutefois, quelques heures plus tard, elle est devenue anémique. Une échographie abdominale a alors été réalisée et a permis de dépister un hématome intra-abdominal. Sur la base de ces observations, la décision de transfuser et de réaliser une exploration chirurgicale de l'abdomen a été prise. En période postopératoire, la patiente est devenue hypoxique, et une échographie thoracique de contrôle a révélé la présence de queues de comètes (des artéfacts échographiques équivalant aux lignes B de Kerley), suggérant un syndrome alvéolaire interstitiel. L'absence d'une pression de remplissage élevée à l'échographie cardiaque suggérait un œdème pulmonaire non cardiogénique et la possibilité d'un syndrome de détresse respiratoire aiguë.

L'autre cas décrit ${ }^{2}$ est celui d'un homme de 58 ans devant subir une excision de polypes nasaux en chirurgie non urgente. Le patient avait une sténose aortique mais un cardiologue et un chirurgien cardiaque l'ont déclaré prêt pour la chirurgie. L'évaluation anesthésique préopératoire de la fonction cardiaque, réalisée par échographie au chevet, a révélé un important épanchement péricardique non soupçonné accompagné de signes de tamponnade péricardique et de collapsus de l'oreillette et du ventricule droits. L'opération a été annulée et le patient a subi un drainage péricardique percutané échoguidé. Les signes cliniques et l'analyse du liquide péricardique ont permis de poser un diagnostic de syndrome de Churg-Strauss, compliqué d'un épanchement péricardique progressif. Le patient a reçu son congé de l'hôpital plusieurs jours plus tard, et ses polypes nasaux ont été traités médicalement.

Ces deux cas illustrent bien l'intégration progressive de l'échographie ciblée comme examen au chevet réalisé par l'anesthésiologiste pour dépister des pathologies qui ne sont pas inhabituelles dans la période périopératoire. ${ }^{5}$ L'échographie a été utilisée dans le cadre d'une approche orientée vers un objectif où ciblé, c'est-à-dire répondre à une question spécifique. De plus, cet examen ne se limitait pas uniquement au cœur. En tant qu'anesthésiologistes et intensivistes, nous nous efforçons de maintenir un transport d'oxygène adéquat. L'échographie peut jouer un rôle dans l'identification de situations qui pourraient entraver l'oxygénation tissulaire, comme l'instabilité hémodynamique, l'hypoxémie et une baisse du débit urinaire.

\section{L'instabilité hémodynamique}

L'échographie au chevet permet de diagnostiquer rapidement toute instabilité hémodynamique et de répéter l'examen au besoin afin d'évaluer la réponse au traitement ou d'exclure une autre pathologie. Le débit cardiaque ne dépend pas exclusivement des pressions de remplissage. Selon le concept mis au point par Guyton pour expliquer les états de choc, le débit cardiaque est, à l'état d'équilibre, égal au retour veineux, lequel est déterminé par trois variables: la tension veineuse systémique moyenne, la pression auriculaire droite et la résistance au retour veineux. ${ }^{6}$ Par conséquent, l'instabilité hémodynamique est le résultat de l'un de ces trois mécanismes distincts, ou d'une combinaison de ces trois mécanismes. L'échographie au chevet permet d'identifier le ou les mécanismes en question. Notre tableau classe les résultats échocardiographiques des deux présentations de cas $^{1,2}$ selon les mécanismes impliqués. La pression veineuse systémique moyenne de la première patiente ${ }^{1}$ était d'abord réduite, signe d'un choc distributif ou hypovolémique dû à une hémorragie. Le second patient, ${ }^{2}$ par contre, présentait une dysfonction systolique et diastolique du ventricule gauche combinée à une tamponnade, ce qui a provoqué une augmentation de la pression auriculaire droite. Une résistance au retour veineux a été observée dans les deux cas. Chez la patiente souffrant de pré-éclampsie, une compression extrinsèque et l'apparition d'un syndrome du 
Tableau Rôle de l'échographie au chevet pour l'identification des variables impliquées dans l'apport d'oxygène

\begin{tabular}{|c|c|c|c|}
\hline $\begin{array}{l}\text { Contenu artériel } \\
\text { d'oxygène }\end{array}$ & $\begin{array}{l}\text { a-Hémoglobine réduite: } \\
\text { détection de liquide dans la } \\
\text { cavité abdominale, } \\
\text { compatible avec un } \\
\text { saignement. }{ }^{1}\end{array}$ & $\begin{array}{l}\text { b-Saturation en oxygène réduite: } \\
\text { Lignes B ou artéfacts en queues } \\
\text { de comètes, compatible avec un } \\
\text { œdème pulmonaire }{ }^{1}\end{array}$ & \\
\hline $\begin{array}{l}\text { Débit cardiaque ou } \\
\text { retour veineux }\end{array}$ & $\begin{array}{l}\text { a-Pression systémique veineuse } \\
\text { moyenne basse: détection de } \\
\text { liquide dans la cavité } \\
\text { abdominale, compatible } \\
\text { avec un volume réduit. }{ }^{1}\end{array}$ & $\begin{array}{l}\text { b-Pression auriculaire droite } \\
\text { accrue: dysfonctionnement } \\
\text { systolique et diastolique du } \\
\text { ventricule gauche }^{1} \text { et sténose } \\
\text { aortique }^{2}\end{array}$ & $\begin{array}{l}\text { c-Résistance au retour } \\
\text { veineux: syndrome du } \\
\text { compartiment en raison } \\
\text { d'un hématome utérin }{ }^{1} \text { et } \\
\text { de tamponnade } \\
\text { péricardique }^{2}\end{array}$ \\
\hline
\end{tabular}

compartiment abdominal ${ }^{7}$ en raison d'un hématome utérin ont provoqué une instabilité hémodynamique et un choc obstructif. Chez le patient atteint d'épanchement péricardique, le choc obstructif était dû à une tamponnade cardiaque. Plusieurs des maladies décrites ci-dessus n'auraient pas pu être diagnostiquées en se fondant exclusivement sur les pressions de remplissage. L'échographie était le meilleur outil de monitorage et de diagnostic dans ces deux cas potentiellement fatals.

\section{L'hypoxémie}

L'échographie au chevet peut être utilisée pour déterminer l'étiologie de l'hypoxémie dans plus de $90 \%$ des cas. ${ }^{8}$ L'utilisation de l'échographie pulmonaire par les anesthésiologistes en salle d'opération a été résumée dans un éditorial récent, ${ }^{9}$ mais la technique n'est pas nouvelle; en effet, il y a vingt ans déjà, elle avait été décrite en détail dans la littérature des soins intensifs. ${ }^{10}$ Bien que l'utilité de l'échographie pulmonaire ne soit plus à démontrer en médecine d'urgence, elle n'est que peu enseignée dans nos programmes de formation d'anesthésiologie ou de soins intensifs. Les détails concernant l'utilisation de cette technique sont disponibles dans deux articles de synthèse clés $^{11,12}$ et dépassent le propos de cet éditorial. Toutefois, en comprenant les mécanismes sous-jacents à l'hypoxémie, il est plus aisé de prendre conscience des limites de l'échographie pulmonaire. En résumé, il existe six mécanismes d'hypoxémie ou de désaturation: un shunt pulmonaire ou extrapulmonaire; des anomalies des rapports ventilation/perfusion; une diminution de la diffusion; une faible fraction d'oxygène inspiré; une désaturation en oxygène du sang veineux mêlé; et une hypoventilation. ${ }^{13}$ Chez la première patiente, les auteurs ont tout d'abord mentionné la présence d'un profil pulmonaire normal avec des lignes A et un glissement pleural (la plèvre viscérale se déplaçant sur la plèvre pariétale). Le glissement pleural permet d'exclure un diagnostic de pneumothorax. Par la suite, on a observé des lignes B ou artéfacts en «queues de comète ». Ces lignes B sont produites par l'interface liquide - air; elles sont le signe d'un syndrome alvéolaire interstitiel et pourraient être dues à un œdème pulmonaire cardiogénique ou non cardiogénique. C'est pourquoi il est essentiel d'analyser les résultats échographiques cardiaques et pulmonaires combinés afin d'exclure l'une ou l'autre étiologie. Les pressions de remplissage ont été estimées par l'utilisation du Doppler et on a d'abord observé qu'elles se situaient dans la norme, lorsque des lignes A étaient visibles. Par la suite, les pressions de remplissage ont augmenté, et des lignes B sont apparues; cela pouvait indiquer un dysfonctionnement systolique et diastolique du ventricule gauche ou être une manifestation précoce d'un syndrome de détresse respiratoire aiguë. Les mécanismes de l'hypoxie ne peuvent pas tous être dépistés grâce à l'échographie pulmonaire et certains cas, tels que le shunt intracardiaque, pourraient également nécessiter une échographie cardiaque. En outre, l'hypoxie due à une faible fraction d'oxygène inspiré ou à une hypoventilation se dépiste grâce à une observation clinique vigilante plutôt qu'à un examen échographique réalisé par un expert.

\section{La baisse du débit urinaire}

Le débit urinaire peut diminuer en raison de causes prérénales, rénales ou postrénales, ou d'une combinaison de ces facteurs. Les problèmes postrénaux peuvent être examinés au chevet en obtenant des images des reins et de la vessie afin d'exclure l'hydronéphrose ou une distension de la vessie due au mauvais positionnement ou à une déformation d'un cathéter urinaire. Les problèmes prérénaux sont évalués en examinant les causes de l'instabilité hémodynamique, tout comme c'était le cas chez la première patiente. ${ }^{1}$ Les causes rénales, comme la néphrite interstitielle, la glomérulonéphrite et la nécrose tubulaire aiguë, pourraient ne pas être dépistées par l'échographie. L'hypertension abdominale est une autre cause importante de baisse du débit urinaire qui passe 
souvent inaperçue; celle-ci peut être due à un syndrome du compartiment abdominal, ${ }^{7}$ comme c'était le cas chez la première patiente, qui a développé un important hématome intra-abdominal. ${ }^{1}$

\section{L'évaluation préopératoire}

Grâce à l'adoption de plus en plus répandue de dispositifs portatifs, l'échographie sera progressivement intégrée et deviendra un prolongement naturel de l'anamnèse du patient et de l'examen physique, comme l'illustre le cas du patient présentant des antécédents de sténose aortique. ${ }^{2}$ Dans ce cas particulier, l'examen échographique a révélé un épanchement péricardique insoupçonné. L'échographie a été utilisée comme outil complémentaire pendant l'évaluation préopératoire, non seulement pour évaluer la fonction cardiaque, mais également pour dépister l'œdème pulmonaire, identifier un épanchement pleural, examiner les voies aériennes, ${ }^{14}$ évaluer le contenu de l'estomac, ${ }^{15}$ évaluer les mouvements du diaphragme, ${ }^{16}$ et exclure une thrombose veineuse profonde. ${ }^{17}$ Dans une étude rétrospective, un groupe basé à Melbourne en Australie a montré que les patients admis avec des fractures de la hanche et qui avaient subi un examen échographique transthoracique ciblé affichaient une mortalité plus faible que leur groupe témoin. ${ }^{18}$ Des études prospectives devraient être réalisées pour confirmer ces résultats dans des cadres semblables.

\section{Limites et problèmes de formation}

L'échographie au chevet doit compléter, et non remplacer, le jugement clinique; dès lors, cette technique comporte des limites. Lorsque l'opérateur concentre son attention sur les images échographiques, des informations plus élémentaires, telles que la pression artérielle et la saturation en oxygène, ne devraient pas pour autant être négligées. En outre, il n'y a pas d'alarme sur un appareil d'échographie, et l'interprétation d'images dépend de l'opérateur. C'est pourquoi l'utilité et l'impact de cet outil dépendent non seulement de la qualité de la formation, mais également du nombre d'examens échographiques réalisés pendant la formation. De nombreuses sociétés ont mis au point des directives de formation pour garantir la compétence de leurs membres. Dans le domaine des soins critiques, l'American College of Chest Physicians et la Société de réanimation de langue française ont publié de telles directives en 2009. ${ }^{19}$ Le premier examen d'échographie en soins critiques a suivi en 2011. Dans un compte rendu récent de 2012, on suggère d'étendre les examens échographiques au chevet à de nombreuses situations en anesthésiologie. ${ }^{20}$ À ce jour, la plupart des directives ont été mises au point à l'intention des cardiologues, des médecins urgentistes, des anesthésiologistes cardiaques et des intensivistes. Il n'existe pas de directive de formation claire à l'intention des anesthésiologistes non cardiaques. Au Canada, la Société canadienne d'échographie au département d'urgence (SCÉDU) (www.scedu.ca) offre une accréditation de formation principalement aux médecins urgentistes. En Ontario, les praticiens doivent être accrédités par la SCÉDU à des fins de facturation. La Section cardiovasculaire et thoracique de la Société canadienne des anesthésiologistes travaille actuellement à la mise au point d'un programme de formation via le Collège royal pour régler la question d'accréditation en surspécialité pour les stagiaires (fellows) en anesthésie cardiaque. Notre groupe, composé des Sections cardiovasculaire et thoracique, de médecine périopératoire et d'échocardiographie périopératoire, collabore afin de mettre au point des directives d'échocardiographie périopératoire dans le cadre non cardiaque. En anesthésiologie, le besoin d'une formation adaptée, d'accréditation et de formation pour les niveaux de base et avancé va devenir pressant dans les années à venir.

Des anesthésiologistes de l'Université de Melbourne ont mis au point des cours en ligne menant à une accréditation en échographie au chevet du patient. Étant donné l'augmentation de la demande pour cette technologie, la formation en échographie devrait peut-être être envisagée plus tôt dans la formation en anesthésie. Plusieurs écoles de médecine offrent désormais un curriculum d'échographie durant le premier cycle d'études. ${ }^{21}$ Aujourd'hui, dans de nombreuses unités de soins intensifs dans le monde, les décisions au chevet se prennent souvent sur la base d'un examen physique échoguidé, ce qui n'était pas le cas il y a dix ans. ${ }^{22}$ L'échographie est déjà utilisée en salle d'opération pour dépister les causes de l'instabilité hémodynamique, de l'hypoxémie, et de l'oligurie/anurie. Grâce à la miniaturisation et à son coût réduit, son utilisation sera bientôt répandue à toutes les salles d'opération. C'est pourquoi des directives pour l'échographie périopératoire hors de la salle d'opération cardiaque sont nécessaires. La prochaine étape logique est de nous former et d'enseigner à nos résidents l'utilisation de cette technologie pour les cas urgents et non urgents; de nombreuses études portant sur les applications de cette technologie apparaîtront à l'avenir. Un parallèle peut être fait avec l'accès vasculaire échoguidé. Il y a quelques années, cette technique semblait être une variante facultative séduisante pour les technophiles. Aujourd'hui, de multiples études ont montré que l'accès vasculaire échoguidé affichait un taux de réussite plus élevé que la technique traditionnelle se fondant sur des repères 
anatomiques; il entraîne moins de complications et prend moins de temps. ${ }^{23}$ À ce jour, les avantages de l'échographie au chevet n'ont pas été établis avec une telle qualité de données probantes. L'une des raisons de cette lacune est que l'impact de cette technique n'est documenté que dans des présentations de cas telles que celles publiées dans ce numéro du Journal. Il est important que l'impact et les limites de cette nouvelle approche soient évalués dans des études cliniques d'envergure bien conçues, étant donné que l'échographie devient à la fois un ajout à la machine d'anesthésie et un prolongement de l'examen physique.

Acknowledgements The authors sincerely thank Denis Babin MSc and Tina Louise Boivin for their help with the editorial.

Funding source Fonds de la Recherche en Santé du Québec.

Competing interests None declared.

Source de financement Fonds de la Recherche en Santé du Québec.

Conflits d'intérêt Aucun.

\section{References}

1. Zieleskiewicz L, Pierrou C, Ragonnet B, et al. Pré-éclampsie sévère et hémorragie post-partum: apport de l'échographie «corps entier ». Can J Anesth 2013; 60. DOI:10.1007/s12630-013-9967-x.

2. Gerlach RM, Saha TK, Allard RV, Tanzola RC. Unrecognized tamponade diagnosed on pre-induction by focused echocardiography. Can J Anesth 2013; 60. DOI:10.1007/s12630013-9968-9.

3. Tanzola RC, Walsh S, Hopman WM, Sydor D, Arellano R, Allard $R V$. Brief report: Focused transthoracic echocardiography training in a cohort of Canadian anesthesiology residents: a pilot study. Can J Anesth 2013; 60: 32-7.

4. Nagueh SF, Middleton KJ, Kopelen HA, Zoghbi WA, Quinones $M A$. Doppler tissue imaging: a noninvasive technique for evaluation of left ventricular relaxation and estimation of filling pressures. J Am Coll Cardiol 1997; 30: 1527-33.

5. Moore CL, Copel JA. Point-of-care ultrasonography. N Engl J Med 2011; 364: 749-57.

6. Funk DJ, Jacobsohn E, Kumar A. The role of venous return in critical illness and shock-part I: physiology. Crit Care Med 2013; 41: 255-62.

7. Deslauriers N, Dery $R$, Denault A. Acute abdominal compartment syndrome. Can J Anesth 2009; 56: 678-82.

8. Lichtenstein DA, Meziere GA. Relevance of lung ultrasound in the diagnosis of acute respiratory failure: the BLUE protocol. Chest 2008; 134: 117-25.
9. Johnson DW, Oren-Grinberg A. Perioperative point-of-care ultrasonography: the past and the future are in anesthesiologists' hands. Anesthesiology 2011; 115: 460-2.

10. Lichtenstein $D$, Axler $O$. Intensive use of general ultrasound in the intensive care unit. Prospective study of 150 consecutive patients. Intensive Care Med 1993; 19: 353-5.

11. Volpicelli $G$, Elbarbary $M$, Blaivas $M$, et al. International evidence-based recommendations for point-of-care lung ultrasound. Intensive Care Med 2012; 38: 577-91.

12. Piette E, Daoust R, Denault A. Basic concepts in the use of thoracic and lung ultrasound. Curr Opin Anaesthesiol 2013; 26: 20-30.

13. Denault AY, Carrier FM. Évaluation de la détresse respiratoire et définition des concepts de base de son traitement. In: Guay J, Martin R, Plaud B, et al., (Eds). Précis d'Anesthésie et de Réanimation, Cinquième Édition. Montreal: Les Presses de l'Université de Montréal; 2012: 118-36.

14. Wojtczak JA. Submandibular sonography: assessment of hyomental distances and ratio, tongue size, and floor of the mouth musculature using portable sonography. J Ultrasound Med 2012; 31: 523-8.

15. Bouvet L, Mazoit JX, Chassard D, Allaouchiche B, Boselli E, Benhamou D. Clinical assessment of the ultrasonographic measurement of antral area for estimating preoperative gastric content and volume. Anesthesiology 2011; 114: 1086-92.

16. Kim SH, Na S, Choi JS, Na SH, Shin S, Koh SO. An evaluation of diaphragmatic movement by M-mode sonography as a predictor of pulmonary dysfunction after upper abdominal surgery. Anesth Analg 2010; 110: 1349-54.

17. Kaabachi O, Alkaissi A, Koubaa W, Aloui N, Toumi NH. Screening for deep venous thrombosis after idiopathic scoliosis surgery in children: a pilot study. Paediatr Anaesth 2010; 20: 144-9.

18. Canty DJ, Royse CF, Kilpatrick D, Bowyer A, Royse AG. The impact on cardiac diagnosis and mortality of focused transthoracic echocardiography in hip fracture surgery patients with increased risk of cardiac disease: a retrospective cohort study. Anaesthesia 2012; 67: 1202-9.

19. Mayo PH, Beaulieu Y, Doelken P, et al. American College of Chest Physicians/La Société de Réanimation de Langue Francaise statement on competence in critical care ultrasonography. Chest 2009; 135: 1050-60.

20. Royse CF, Canty DJ, Faris J, Haji DL, Veltman M, Royse A. Core review: physician-performed ultrasound: the time has come for routine use in acute care medicine. Anesth Analg 2012; 115: 1007-28.

21. Hoppmann RA, Rao VV, Poston MB, et al. An integrated ultrasound curriculum (iUSC) for medical students: 4-year experience. Crit Ultrasound J 2011; 3: 1-12.

22. Vieillard-Baron A, Slama $M$, Cholley B, Janvier $G$, Vignon P. Echocardiography in the intensive care unit: from evolution to revolution? Intensive Care Med 2008; 34: 243-9.

23. Troianos CA, Hartman GS, Glas KE, et al. Guidelines for performing ultrasound guided vascular cannulation: recommendations of the American Society of Echocardiography and the Society of Cardiovascular Anesthesiologists. J Am Soc Echocardiogr 2011; 24: 1291-318. 